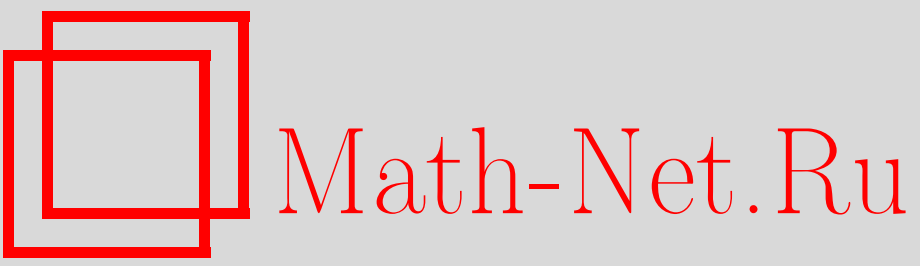

С. С. Постнов, Е. А. Постнова, Об особенностях динамики двумерных линейных систем дробного порядка с управлением, Итоги науки и техн. Сер. Соврем. мат. и ее прил. Темат. обз., 2020, том 182, 101-118

DOI: https://doi.org/10.36535/0233-6723-2020-182-101-118

Использование Общероссийского математического портала Math-Net.Ru подразумевает, что вы прочитали и согласны с пользовательским соглашением

http: //www. mathnet.ru/rus/agreement

Параметры загрузки:

IP: 54.224 .60 .19

26 апреля 2023 г., 02:26:49 


\title{
ОБ ОСОБЕННОСТЯХ ДИНАМИКИ ДВУМЕРНЫХ ЛИНЕЙНЫХ СИСТЕМ ДРОБНОГО ПОРЯДКА С УПРАВЛЕНИЕМ
}

\author{
(c) 2020 г. $\quad$ С. С. Постнов, Е. А. ПостновА
}

\begin{abstract}
АннотАция. Исследуется поведение фазовых траекторий двумерных линейных систем дробного порядка с управлением. Основное внимание уделяется двойному интегратору дробного порядка. Операторы дифференцирования дробного порядка понимаются в смысле Хильфера или Адамара. Допустимые управления считаются ограниченными по норме и ищутся в классе функций из пространства $L_{\infty}[0, T], T>0$. На основе явно заданных ограничений на норму управления вычислены граничные траектории системы, выделяющие на фазовой плоскости область, в которой локализуются все допустимые траектории системы. Демонстрируется, что решение задачи оптимального управления методом моментов приводит к некоторой задаче минимизации, не имеющей аналитического решения в общем случае (при произвольных значениях показателей дробного дифференцирования в уравнениях, описывающих систему). Установлены условия, при которых данная задача минимизации имеет решение и определены подобласти возможной локализации этого решения. Построены точные и приближенные аналитические решения задачи минимизации в некоторых частных случаях, также приведены результаты численного нахождения минимума. Получены соответствующие решения исследуемой задачи оптимального управления, на основе которых вычислены фазовые траектории системы. Проведен анализ полученных результатов.
\end{abstract}

Ключевые слова: фазовая траектория, система дробного порядка, оптимальное управление.

\section{ON THE DYNAMICS OF TWO-DIMENSIONAL FRACTIONAL LINEAR CONTROL SYSTEMS}

\author{
(c) 2020 S. S. POSTNOV, E. A. POSTNOVA
}

\begin{abstract}
In this paper, we examine the behavior of phase trajectories of fractional two-dimensional linear systems with control. We focus on the double fractional integrator. Fractional differentiation operators are understood in the sense of Hilfer or Hadamard. Admissible controls are assumed to be norm-bounded; we search for them in the functional class $L_{\infty}[0, T], T>0$. Based on explicitly specified constraints on the norm of a control, we calculate boundary trajectories of the system, which determine on the phase plane a domain in which all admissible trajectories of the system are localized. We show that the solution of the optimal control problem by the method of moments leads to some minimization problem that does not have an analytical solution in the general case (for arbitrary values of the exponents of fractional differentiation in the equations governing the system). We establish conditions under which the minimization problem considered has a solution and determine subdomains of possible localization of this solution. Exact and approximate analytical solutions of the minimization problem are constructed in some particular cases and the results of numerical computation of the minimum are given. The corresponding solutions of the optimal control problem are obtained and phase trajectories of the system are found. All results obtained are analyzed.
\end{abstract}

Keywords and phrases: phase trajectory, fractional order system, optimal control.

AMS Subject Classification: 49N05, 49J21, 93C23, 34K35, 34A08 
1. Введение. Исследования систем дробного порядка с управлением являются относительно новым направлением дробной динамики и теории дифференциальных уравнений дробного порядка, активно развивающимся последние 10-15 лет. При этом важной особенностью систем дробного порядка является зависимость их свойств и поведения от выбранного определения оператора дробного дифференцирования, которых в настоящее время насчитывается уже несколько десятков. Математический аппарат дробного исчисления активно используется для моделирования самых разных систем: с помощью интегро-дифференциальных операторов дробного порядка описывается связь между силой и скоростью или ускорением объекта, подверженного ее действию, связь между током в цепи и напряжением на обкладках конденсатора с сильно-неоднородным заполнителем и т. п. В относительно простых моделях систем с управлением (роль которого может играть внешняя сила, электрическое или механическое напряжение, деформация и т. п.) упомянутая связь может быть представлена моделью одиночного или двойного дробного интегратора, обобщающей обычный интегратор целого порядка на случай, когда соответствующий оператор интегрирования/дифференцирования имеет произвольный дробный порядок.

Первыми работами, в которых рассматривались модели одиночного и двойного интеграторов, были работы $[5,6,14,16]$, в которых обсуждались среди прочего модели управления движением. Затем двойной и $N$-кратный интеграторы рассматривались в работах по моделированию поведения многоагентных систем (см. [10]) и построению алгоритмов стабилизации систем дробного порядка в условиях неопределенности (см. [12]). В этих работах используется определение дробного оператора в смысле Капуто (см. [13]). В настоящей работе рассматриваются задачи оптимального управления $N$-кратным интегратором (при $N \leqslant 2$ ) для случаев, когда соответствующий оператор дробного порядка понимается в смысле Хильфера (см. [11]) или Адамара (см. [13]). Исследование проводится для управлений, определяемых существенно ограниченными функциями (т.е. функциями из пространства $\left.u(t) \in L_{\infty}\left(t_{0}, T\right]\right)$. Такие управления представляют значительный интерес как с теоретической, так и с прикладной точки зрения. Они не являются непрерывными и всюду дифференцируемыми функциями, но могут быть получены как точное аналитическое решение задачи оптимального управления с явными ограничениями на норму управления. При этом именно такие функции адекватно моделируют работу реальных технических систем, где управляющее воздействие часто осуществляется с помощью релейных элементов (переключателей).

Полученные в работе результаты обобщают результаты работ [5-9,15], в которых исследовался двойной интегратор дробного порядка. Получены новые точные и приближенные аналитические решения задачи оптимального управления в ряде частных случаев и приведены результаты численных расчетов для общего случая.

2. Постановка задачи. Двойной интегратор представляет собой линейную двумерную стационарную систему следующего вида:

$$
\begin{aligned}
& { }_{t_{0}} D_{t}^{\rho_{1}} q_{1}(t)=q_{2}(t), \\
& { }_{t_{0}} D_{t}^{\rho_{2}} q_{2}(t)=u(t),
\end{aligned}
$$

где функции $q_{1}(t), q_{2}(t)$ и $u(t)$ определяют состояние и управление соответственно, $t \in\left(t_{0}, T\right]$, $T>t_{0}>0$. Управление $u(t)$ считается элементом пространства $L_{\infty}\left(t_{0}, T\right]$. Оператор дробного дифференцирования $t_{0} D_{t}^{\rho_{i}}$ в формуле (1) понимается в смысле Хильфера (см. [11]) или Адамара (см. [13]). Индекс $\rho_{i}$ в первом случае является составным, $\rho_{i}=\left(\alpha_{i}, \mu_{i}\right), \alpha_{i} \in(0,1], \mu_{i} \in[0,1]$, $i=1,2$. При $\mu=0$ и $\mu=1$ оператор Хильфера представляет собой соответственно оператор Римана-Лиувилля и Капуто. Во втором случае $\rho_{i}=\alpha_{i} \in(0,1]$. Будем далее говорить в этих двух случаях о системе (двойном интеграторе) Хильфера или Адамара соответственно.

Начальные условия для системы (1) задаются в виде

$$
\lim _{t \rightarrow t_{0}+}\left[t_{0} I_{t}^{\sigma_{i}} q_{i}(t)\right]=s_{i}^{0}, \quad i=1,2,
$$


где оператор дробного интегрирования $t_{0} I_{t}^{\sigma_{i}}$ понимается следующим образом: в смысле РиманаЛиувилля (см. [13]) с показателем $\sigma_{i}=\left(1-\alpha_{i}\right)\left(1-\mu_{i}\right)$, если оператор дробного дифференцирования в уравнениях (1) понимается в смысле Хильфера и в смысле Адамара с показателем $\sigma_{i}=\alpha_{i}$, если оператор дробного дифференцирования в уравнениях (1) понимается в смысле Адамара.

Конечные условия для системы (1) задаются в виде

$$
q_{i}(T)=q_{i}^{T}, \quad i=1,2 .
$$

Замечание 1. Вообще говоря, начальные и конечные условия вида (2) и (3) можно рассматривать и в параметрическом виде, учитывая возможную зависимость величин в правой части от выбора начального и конечного моментов времени (см. [9]).

Задача оптимального управления формулируется следующим образом: найти управление $u(t)$, переводящее систему (1) из заданного начального состояния, определяемого условиями (2), в заданное конечное состояние (3), и имеющее минимальную норму $\|u(t)\|$ при заданном времени управления $T-t_{0}$ (времени перехода системы из начального состояния в конечное). Здесь $\|u(t)\|=$ vrai $\max _{t} \in\left(t_{0}, T\right] u(t)$.

Можно показать (см. [5-8]), что поставленная задача оптимального управления для исследуемой системы (1) сводится к следующей $l$-проблеме моментов (см. $[1,3])$ : построить такую функцию $u(t) \in L_{\infty}\left(t_{0}, T\right]$, что выполняются соотношения

$$
\int_{t_{0}}^{T} g_{n}(\tau) u(\tau) d \tau=c_{n}, \quad n=1,2,
$$

и условие

$$
\|u(t)\| \leqslant l,
$$

где $g_{n}(\tau) \in L_{1}\left(t_{0}, T\right]$ - некоторые функции, $c_{n}$ - некоторые числа (моменты), хотя бы одно из которых отлично от нуля. В случае системы Хильфера имеют место следующие выражения:

$$
\begin{gathered}
g_{1}(\tau)=\frac{(T-t)^{\alpha_{1}+\alpha_{2}-1}}{\Gamma\left(\alpha_{1}+\alpha_{2}\right)}, \quad g_{2}(\tau)=\frac{(T-t)^{\alpha_{2}-1}}{\Gamma\left(\alpha_{2}\right)}, \\
c_{1}=q_{1}^{T}-\frac{s_{1}^{0}\left(T-t_{0}\right)^{-\left(1-\mu_{1}\right)\left(1-\alpha_{1}\right)}}{\Gamma\left[1-\left(1-\mu_{1}\right)\left(1-\alpha_{1}\right)\right]}-\frac{s_{2}^{0}\left(T-t_{0}\right)^{\alpha_{1}-\left(1-\mu_{2}\right)\left(1-\alpha_{2}\right)}}{\Gamma\left[\alpha_{1}+1-\left(1-\mu_{2}\right)\left(1-\alpha_{2}\right)\right]}, \\
c_{2}=q_{2}^{T}-\frac{s_{2}^{0}\left(T-t_{0}\right)^{-\left(1-\mu_{2}\right)\left(1-\alpha_{2}\right)}}{\Gamma\left[1-\left(1-\mu_{2}\right)\left(1-\alpha_{2}\right)\right]} .
\end{gathered}
$$

Аналогичные величины для системы Адамара выражаются следующим образом:

$$
\begin{gathered}
g_{1}(\tau)=\frac{1}{\Gamma\left(\alpha_{1}+\alpha_{2}\right)}\left(\ln \frac{T}{\tau}\right)^{\alpha_{1}+\alpha_{2}-1} \frac{1}{\tau}, \quad g_{2}(\tau)=\frac{1}{\Gamma\left(\alpha_{2}\right)}\left(\ln \frac{T}{\tau}\right)^{\alpha_{2}-1} \frac{1}{\tau} \\
c_{1}=q_{1}^{T}-\frac{s_{l}^{0}}{\Gamma\left(\alpha_{l}\right)}\left(\ln \frac{T}{t_{0}}\right)^{\alpha_{l}-1}-\frac{s_{2}^{0}}{\Gamma\left(\alpha_{l}+\alpha_{2}\right)}\left(\ln \frac{T}{t_{0}}\right)^{\alpha_{l}+\alpha_{2}-1}, \\
c_{2}=q_{2}^{T}-\frac{s_{2}^{0}}{\Gamma\left(\alpha_{2}\right)}\left(\ln \frac{T}{t_{0}}\right)^{\alpha_{2}-1} .
\end{gathered}
$$

В свою очередь, $l$-проблема моментов (4)-(5) для системы (1) сводится к задаче поиска минимума следующей функции (см. [5-8]):

$$
F\left(\xi_{2}\right)=\int_{t_{0}}^{T}\left|\frac{1-c_{2} \xi_{2}}{c_{1}} g_{1}(\tau)+\xi_{2} g_{2}(\tau)\right| d \tau
$$

где $\xi_{2} \in(-\infty, \infty)$ - действительная переменная. Очевидно, функция $F\left(\xi_{2}\right)$ положительно определена по построению. Значение функции (12) в точке минимума $\xi_{2}^{*}$ определяет величину нормы 
оптимального управления (константы, обозначаемой в литературе $\lambda_{2}$ ) (см. $\left.[1,3]\right)$ :

$$
F\left(\xi_{2}^{*}\right)=\frac{1}{\lambda_{2}}
$$

Введем обозначение

$$
f\left(\tau, \xi_{2}\right)=\frac{1-c_{2} \xi_{2}}{c_{1}} g_{1}(\tau)+\xi_{2} g_{2}(\tau)
$$

С помощью этой функции строится решение поставленной задачи оптимального управления (см. $[1,3])$ :

$$
u(t)=\lambda_{2} \operatorname{sign}\left[f\left(t, \xi_{2}^{*}\right)\right]
$$

Функция (8), стоящая под знаком модуля в интеграле в формуле (6), может менять знак в некоторой точке $t^{\prime}$, которая выражается следующими формулами:

$$
t^{\prime}=T-\left(\frac{c_{1} \xi_{2}}{c_{2} \xi_{2}-1} \frac{\Gamma\left(\alpha_{1}+\alpha_{2}\right)}{\Gamma\left(\alpha_{2}\right)}\right)^{\frac{1}{\alpha_{1}}}
$$

для системы Хильфера и

$$
t^{\prime}=T \exp \left[-\left(\frac{c_{1} \xi_{2}}{c_{2} \xi_{2}-1} \frac{\Gamma\left(\alpha_{1}+\alpha_{2}\right)}{\Gamma\left(\alpha_{2}\right)}\right)^{\frac{1}{\alpha_{1}}}\right]
$$

для системы Адамара. Случай, когда упомянутая функция не меняет знака, не представляет существенного интереса и далее рассматриваться не будет.

Вычисление интеграла в формуле (6) приводит к выражению вида

$$
F\left(\xi_{2}\right)=\left\{\begin{array}{l}
\left(A\left(\frac{c_{1} \xi_{2}}{c_{2} \xi_{2}-1}\right)^{\frac{\alpha_{2}}{\alpha_{1}}} \xi_{2}+B \frac{c_{2} \xi_{2}-1}{c_{1}}-C \xi_{2}\right) \operatorname{sign}\left(\xi_{2}\right), \quad \xi_{2} \neq 0 \\
\frac{B}{\left|c_{1}\right|}, \quad \xi_{2}=0
\end{array}\right.
$$

где

$$
\begin{aligned}
& A=\frac{2 \alpha_{1}}{\left(\alpha_{1}+\alpha_{2}\right) \Gamma\left(\alpha_{1}+\alpha_{2}\right)}\left[\frac{\Gamma\left(\alpha_{1}+\alpha_{2}\right)}{\Gamma\left(\alpha_{2}\right)}\right]^{\frac{\alpha_{2}}{\alpha_{1}}}, \\
& B= \begin{cases}\frac{T^{\alpha_{1}+\alpha_{2}}}{\Gamma\left(\alpha_{1}+\alpha_{2}+1\right)} & \text { в случае системы Хильфера, } \\
\frac{\left(\ln \frac{T}{t_{0}}\right)^{\alpha_{1}+\alpha_{2}}}{\Gamma\left(\alpha_{1}+\alpha_{2}+1\right)} & \text { в случае системы Адамара, }\end{cases} \\
& C= \begin{cases}\frac{T^{\alpha_{2}}}{\Gamma\left(\alpha_{2}+1\right)} & \text { в случае системы Хильфера, } \\
\frac{\left(\ln \frac{T}{t_{0}}\right)^{\alpha_{2}}}{\Gamma\left(\alpha_{2}+1\right)} & \text { в случае системы Адамара. }\end{cases}
\end{aligned}
$$

3. Исследование задачи минимизации функции $F\left(\xi_{2}\right)$. Далее будут сформулированы условия, при которых функция (12) имеет минимум, и указаны подобласти области определения данной функции, в которых могут лежать точки минимума.

Теорема 1. Функиия $F\left(\xi_{2}\right)$, определяемая формулой (12), достигает своего минимума либо в стационарных точках, либо при $\xi_{2}=0$. 
Доказательство. Как известно, произвольная функция может достигать своих экстремальных значений либо в стационарных точках, либо в точках, относящихся к границе области определения, либо в точках, где производная этой функции не определена. Непосредственным вычислением из формулы (12) можно получить следующую асимптотику:

$$
F\left(\xi_{2}\right) \sim O\left(\xi_{2}\right), \quad F\left(\xi_{2}\right) \rightarrow \infty \quad \text { при } \xi_{2} \rightarrow \pm \infty .
$$

Следовательно, достигать минимума на границах области определения функция (12) не может.

Функция (12) имеет разрывы при $\xi_{2}=1 / c_{2}$ и при $\xi_{2}=0$. При $\xi_{2}=1 / c_{2}$ и функция, и ее производная обращаются в бесконечность, поэтому эта точка не может быть точкой минимума функции. В точке же $\xi_{2}=0$ функция (12) определена, а ее производная - нет. Потенциально данная точка может быть точкой минимума рассматриваемой функции.

Наконец, функция (12) ограничена, непрерывна и бесконечно дифференцируема почти всюду на вещественной оси (за исключением двух вышеупомянутых точек разрыва) и, следовательно, имеет стационарные точки, в которых может достигать своих экстремальных значений.

Таким образом, функция (12) может достигать минимума либо в своих стационарных точках, либо в точке $\xi_{2}=0$.

Теорема 2. Функиия (12) может достигать минимума только в таких точках $\xi_{2} \in$ $(-\infty, \infty)$, для которых одновременно выполняются следующие условия:

$$
\text { (i) } c_{2} \xi_{2} \neq 1 ; \quad \text { (ii) } \operatorname{Im}\left(\frac{c_{1} \xi_{2}}{c_{2} \xi_{2}-1}\right)^{\frac{\alpha_{2}}{\alpha_{1}}}=0 ; \quad \text { (iii) } F\left(\xi_{2}\right)>0 .
$$

Доказательство. Если не выполнено первое условие, т.е. $c_{2} \xi_{2}=1$, то точка $\xi_{2}$ является точкой разрыва функции (12). В этой точке, как следует непосредственно из формулы (12), функция обращается в бесконечность и, следовательно, не может принимать минимального значения. Если не выполнено второе и/или третье условие, то функция (12) не является вещественнозначной и положительно определенной, что противоречит свойствам, которые данная функция должна иметь по построению (исходя из формулы (6)). Таким образом, точки $\xi_{2}$, для которых не выполнено хотя бы одно из условий теоремы, не могут быть точками минимума функции (12).

Замечание 2. Условие (ii) в теореме 2, как можно убедиться с помощью формул (10) и (11), равносильно условию вещественности значения момента времени $t^{\prime}$. Если дополнительно полагать, что выполняется условие $t^{\prime}<T$ (что соответствует рассматриваемому случаю, когда функция (8) меняет знак на интервале $t \in\left(t_{0}, T\right)$ ), то условие (ii) в теореме 2 можно заменить условием:

$$
\frac{c_{1} \xi_{2}}{c_{2} \xi_{2}-1}>0 \text {. }
$$

Теорема 3. Пусть выполнены условия теорем 1 и 2 и функиия, определяемая формулой (8), меняет знак на интервале $t \in\left(t_{0}, T\right)$. Тогда допустимые точки минимума функиии (12) могут лежать только на следующих интервалах:

$$
\begin{array}{ll}
\xi_{2} \in\left(\frac{1}{c_{2}-k c_{1}}, \frac{1}{c_{2}}\right) & \text { npu } c_{1}<0, c_{2}>0, \\
\xi_{2} \in\left(\frac{1}{c_{2}-k c_{1}}, 0\right) & \text { npu } c_{1}>0, c_{2}<0, \\
\xi_{2} \in(-\infty, 0) \cup\left(\frac{1}{c_{2}-k c_{1}}, \infty\right) & \text { npu } c_{1}>0, c_{2}>0, c_{2}-k c_{1}>0, \\
\xi_{2} \in\left(\frac{1}{c_{2}-k c_{1}}, 0\right) & \text { npu } c_{1}>0, c_{2}>0, c_{2}-k c_{1}<0, \\
\xi_{2} \in\left(-\infty, 1 / c_{2}\right) \cup(0, \infty) & \text { npu } c_{1}<0, c_{2}<0, c_{2}-k c_{1}<0, \\
\xi_{2} \in\left(0, \frac{1}{c_{2}-k c_{1}}\right) & \text { npu } c_{1}<0, c_{2}<0, c_{2}-k c_{1}>0,
\end{array}
$$


где

$$
k= \begin{cases}\frac{\Gamma\left(\alpha_{1}+\alpha_{2}\right)}{\Gamma\left(\alpha_{2}\right)}\left(T-t_{0}\right)^{-\alpha_{1}} & \text { для системъ Хильфера, } \\ \frac{\Gamma\left(\alpha_{1}+\alpha_{2}\right)}{\Gamma\left(\alpha_{2}\right)}\left(\ln \frac{T}{t_{0}}\right)^{-\alpha_{1}} & \text { для системъ Адамара. }\end{cases}
$$

Доказательство. В случае, когда функция, определяемая формулой (8), меняет знак на интервале $t \in\left(t_{0}, T\right)$, имеют место неравенства: $0<t_{0}<t^{\prime}<T$, где $t^{\prime}$ определяется с помощью формул (10) и (11).

Рассмотрим сначала случай $t^{\prime}<T$. Тогда, как указано выше, второе условие теоремы 2 эквивалентно условию (13). Отсюда, в частности, следует, что $\xi_{2} \neq 0$. Неравенство (13) будет выполняться, когда и числитель, и знаменатель в левой части имеют один знак. Решив соответствующую систему неравенств, можно получить условия:

$$
\begin{array}{ll}
\xi_{2} \in\left(0, \frac{1}{c_{2}}\right) & \text { при } c_{1}<0, c_{2}>0, \\
\xi_{2} \in\left(\frac{1}{c_{2}}, 0\right) & \text { при } c_{1}>0, c_{2}<0, \\
\xi_{2} \in(-\infty, 0) \cup\left(\frac{1}{c_{2}}, \infty\right) & \text { при } c_{1}>0, c_{2}>0, \\
\xi_{2} \in\left(-\infty, \frac{1}{c_{2}}\right) \cup(0, \infty) & \text { при } c_{1}<0, c_{2}<0,
\end{array}
$$

Если теперь использовать условие $t^{\prime}>t_{0}$, то с помощью формул (10) и (11) можно составить еще одну систему неравенств, в результате решения которой совместно с системой (15) получаются окончательно условия (14).

4. Точные решения задачи минимизации. Будем рассматривать случай, когда функция, определяемая формулой (8), меняет знак на интервале $t \in\left(t_{0}, T\right)$. Из условия (13) (и также из теоремы 3) следует, что в этом случае минимум не может достигаться при $\xi_{2}=0$. Тогда, согласно теореме 1, единственным типом точек, в которых функция (12) может достигать минимума, являются стационарные точки данной функции. Продифференцировав выражение (12), получим следующее уравнение для поиска таких точек:

$$
\left(\frac{c_{1} \xi_{2}}{c_{2} \xi_{2}-1}\right)^{\alpha_{2} / \alpha_{1}+1} \frac{c_{2} \xi_{2}-1-\alpha_{2} / \alpha_{1}}{c_{1} \xi_{2}}=\frac{1}{A}\left(C-\frac{c_{2}}{c_{1}} B\right)
$$

В общем случае уравнение (16) не имеет точного аналитического решения, т. к. содержит дробно-степенную функцию произвольной (определяемой отношением показателей дробного дифференцирования) степени аргумента. Однако точное решение этого уравнения может быть получено в некоторых частных случаях, из которых мы подробно рассмотрим два: $c_{2}=0$ и $\alpha_{1}=\alpha_{2}=\alpha$.

В случае $c_{2}=0$ функция (12) принимает вид:

$$
F\left(\xi_{2}\right)=\left(A\left(-c_{1}\right)^{\alpha_{2} / \alpha_{1}} \xi_{2}^{\alpha_{2} / \alpha_{1}+1}-C \xi_{2}-\frac{B}{c_{1}}\right) \operatorname{sign}\left(\xi_{2}\right)
$$

Точное решение уравнения (16) в этом случае получено в работах $[7,8,15]$ и приводит к следующему выражению для оптимального управления в случае системы Хильфера:

$$
u(t)=-\frac{\Gamma\left(\alpha_{1}+\alpha_{2}+1\right)}{1-2^{-\frac{\alpha_{1}}{\alpha_{2}}}} \frac{\left|c_{1}\right|}{\left(T-t_{0}\right)^{\alpha_{1}+\alpha_{2}}} \operatorname{sign}\left[\frac{\left(T-t_{0}\right)^{\alpha_{1}}-2^{-\frac{\alpha_{1}}{\alpha_{2}}}\left(T-t_{0}\right)^{\alpha_{1}}}{c_{1}}\right] .
$$

Для системы Адамара имеет место аналогичное выражение:

$$
u(t)=-\frac{\Gamma\left(\alpha_{1}+\alpha_{2}+1\right)}{1-2^{-\frac{\alpha_{1}}{\alpha_{2}}}} \frac{\left|c_{1}\right|}{\left(\ln \frac{T}{t_{0}}\right)^{\alpha_{1}+\alpha_{2}}} \operatorname{sign}\left[\frac{\left(\ln \frac{T}{t}\right)^{\alpha_{1}}-2^{-\frac{\alpha_{1}}{\alpha_{2}}}\left(\ln \frac{T}{t_{0}}\right)^{\alpha_{1}}}{c_{1}}\right], \quad t \in\left(t_{0}, T\right] .
$$


В случае $\alpha_{1}=\alpha_{2}=\alpha$ функция (12) принимает вид:

$$
F\left(\xi_{2}\right)=\left(A \frac{c_{1} \xi_{2}^{2}}{c_{2} \xi_{2}-1}-C \xi_{2}+B \frac{c_{2} \xi_{2}-1}{c_{1}}\right) \operatorname{sign}\left(\xi_{2}\right)
$$

Уравнение (16) при этом сводится к квадратному:

$$
\left(c_{1}-P c_{2}\right) c_{2} \xi_{2}^{2}-2\left(c_{1}-P c_{2}\right) \xi_{2}-P=0,
$$

где

$$
P=\left\{\begin{array}{l}
\frac{\left(T-t_{0}^{\alpha}\right.}{A \Gamma(\alpha+1)}\left(1-\frac{c_{2}}{c_{1}} \frac{\left(T-t_{0}\right)^{\alpha} \Gamma(\alpha+1)}{\Gamma(2 \alpha+1)}\right) \\
\frac{\left(\ln \frac{T}{t_{0}}\right)^{\alpha}}{A \Gamma(\alpha+1)}\left(1-\frac{c_{2}}{c_{1}} \frac{\left(\ln \frac{T}{t_{0}}\right)^{\alpha} \Gamma(\alpha+1)}{\Gamma(2 \alpha+1)}\right)
\end{array}\right.
$$

Данное уравнение имеет вещественные корни при условии:

$$
c_{1}\left(c_{1}-P c_{2}\right) \geqslant 0 \text {. }
$$

Эти корни выражаются следующим образом:

$$
\xi_{2}^{(1,2)}=\frac{1}{c_{2}}\left(1 \pm \sqrt{\frac{c_{1}}{c_{1}-P c_{2}}}\right) .
$$

Тип экстремума функции (12), как известно, можно определить по знаку второй производной, для которой в точках $\xi_{2}=\xi_{2}^{(1,2)}$ будет справедливо выражение:

$$
F^{\prime \prime}\left(\xi_{2}^{(1,2)}\right)= \pm 2 A c_{1}\left(\frac{c_{1}-P c_{2}}{c_{1}}\right)^{3 / 2} \operatorname{sign}\left(\xi_{2}^{(1,2)}\right) .
$$

Соответственно, при заданных значениях параметров выбор корня может осуществляться исходя из условия $F^{\prime \prime}\left(\xi_{2}^{(1,2)}\right)>0$. Этот выбранный корень является точкой $\xi_{2}^{*}$, в которой функция $(12)$ достигает минимума. На основе найденной величины $\xi_{2}^{*}$, в соответствии с формулой (7) определяется норма оптимального управления:

$$
F\left(\xi_{2}^{*}\right)=\left[\frac{A}{c_{2}}\left(1 \pm \sqrt{\frac{c_{1}}{c_{1}-P c_{2}}}\right)\left( \pm \frac{c_{2}}{c_{1}}\left(\sqrt{\frac{c_{1}-P c_{2}}{c_{1}}} \pm 1\right)-P\right)-\frac{B}{c_{1}}\right] \operatorname{sign}\left(\xi_{2}^{*}\right)=\frac{1}{\lambda_{2}} .
$$

Решение задачи оптимального управления (9) в этом случае записывается в виде:

$$
u(t)=\lambda_{2} \operatorname{sign}\left(\mp \sqrt{\frac{c_{1}}{c_{1}-P c_{2}}} \frac{(T-t)^{\alpha}}{c_{1} \Gamma(\alpha)}+\left(1 \pm \sqrt{\frac{c_{1}}{c_{1}-P c_{2}}}\right) \frac{1}{c_{2} \Gamma(2 \alpha)}\right)
$$

для системы Хильфера и

$$
u(t)=\lambda_{2} \operatorname{sign}\left(\mp \sqrt{\frac{c_{1}}{c_{1}-P c_{2}}} \frac{\left(\ln \frac{T}{t}\right)^{\alpha}}{c_{1} \Gamma(\alpha)}+\left(1 \pm \sqrt{\frac{c_{1}}{c_{1}-P c_{2}}}\right) \frac{1}{c_{2} \Gamma(2 \alpha)}\right)
$$

для системы Адамара. Здесь знак «+» соответствует выбору $\xi_{2}^{*}=\xi_{2}^{1}$, а противоположныйвыбору $\xi_{2}^{*}=\xi_{2}^{2}$.

Замечание 3. Управления (17), (18), (19) и (20) являются кусочно-постоянными и имеют одну точку переключения, определяемую формулами (10) и (11). 
5. Приближенные решения задачи минимизации. Как и в предыдущем разделе, будем искать минимум в стационарных точках функции (12). Исследуем теперь возможности приближенного решения уравнения (16) и воспользуемся методами теории возмущений, выбрав в качестве малого параметра либо отношение $c_{2} / c_{1}$, либо отношение $\alpha_{2} / \alpha_{1}$.

Обозначим $c_{2} / c_{1}=x$ и перепишем левую часть равенства (16) в виде функции:

$$
\phi(x)=\left(\frac{c_{1} \xi_{2}}{x c_{1} \xi_{2}-1}\right)^{\alpha_{2} / \alpha_{1}} \frac{x c_{1} \xi_{2}-1-\alpha_{2} / \alpha_{1}}{x c_{1} \xi_{2}-1} .
$$

Рассмотрим теперь случай $c_{2} / c_{1}=x \ll 1$ и разложим левую часть в ряд Тейлора в окрестности точки $x=0$, удержав члены не старше линейного:

$$
\phi(x)=\phi(0)+\phi^{\prime}(0) x+\ldots
$$

Примем при этом:

$$
\xi_{2}=\xi_{2}^{(0)}+\xi_{2}^{(1)} x .
$$

Подставив разложения (21) и (22) в уравнение (16) и выполнив необходимые вычисления, можно получить следующее выражение для оценки значения точки минимума функции (12):

$$
\widehat{\xi}_{2}^{*}=-\frac{1}{c_{1}}\left(\frac{\alpha_{1}}{\alpha_{1}+\alpha_{2}} \frac{C}{A}\right)^{\alpha_{1} / \alpha_{2}}\left[1+\frac{c_{2}}{c_{1}}\left(\frac{\alpha_{1}}{\alpha_{2}} \frac{B}{C}+\frac{2 \alpha_{1}+\alpha_{2}}{\alpha_{1}+\alpha_{2}} \frac{1}{c_{1}}\left(\frac{\alpha_{1}}{\alpha_{1}+\alpha_{2}} \frac{C}{A}\right)^{\alpha_{1} / \alpha_{2}}\right)\right] .
$$

Можно убедиться, что при $x=c_{2} / c_{1}=0$ формула (23) сводится к точной формуле для $\xi_{2}^{*}$, имеющей место при точном решении уравнения (16) в случае $c_{2}=0[6,8,15]$. На основании формулы (23) по формулам (7) и (9) может быть вычислено оптимальное управление и его норма.

Другим случаем, в котором может быть получено приближенное аналитическое решение уравнения (16), является случай $\alpha_{2} / \alpha_{1} \ll 1$. Такой случай может реализовываться, например, когда в первом звене интегратора имеется «классическая» связь $\left(\alpha_{1}=1\right)$, а второе звено является «слабо дифференцирующим», т. е. $\alpha_{2} \sim 0,1 \div 0,2$. При таком допущении левая часть исходного уравнения (16) заметно упрощается, и оно приводится к виду:

$$
\left(\frac{c_{1} \xi_{2}}{c_{2} \xi_{2}-1}\right)^{\alpha_{2} / \alpha_{1}}=\frac{1}{A}\left(C-\frac{c_{2}}{c_{1}} B\right)
$$

Отсюда находим следующую оценку для точки минимума функции (12):

$$
\widehat{\xi}_{2}^{*}=\frac{\left(C c_{1}-B c_{2}\right)^{\alpha_{1} / \alpha_{2}}}{c_{2}\left(C c_{1}-B c_{2}\right)^{\alpha_{1} / \alpha_{2}}-c_{1}^{\alpha_{1} / \alpha_{2}+1} A^{\alpha_{1} / \alpha_{2}}} .
$$

По аналогии с разобранным выше случаем, на основании формулы (24) можно вычислить оптимальное управление (9) и его норму (7).

В случаях, более общих, чем рассмотренные выше, когда нет возможности предполагать малость отношения определенных параметров или заранее выбрать значения некоторых из них, поставленная задача минимизации функции (12) может быть решена численно.

6. Свойства решений задачи оптимального управления. В данном разделе кратко проиллюстрировано поведение нормы оптимального управления в зависимости от показателей дробного дифференцирования в случаях, соответствующих решениям, описанным в предыдущих двух разделах.

На рис. 1 и 2 для системы Капуто приведены зависимости нормы управления от одного из показателей дробного дифференцирования при фиксированном значении второго для случаев $c_{2}=0$ (пунктирные линии) и $c_{2} / c_{1} \ll 1$ (сплошные линии). Были приняты следующие значения параметров: $t_{0}=0, T=10, s_{1}^{0}=10, q_{1}^{T}=0, s_{2}^{0}=0 ; q_{2}^{T}=0$ (в случае $\left.c_{2}=0\right)$ и $q_{2}^{T}=-1$ (в случае $\left.c_{2} / c_{1} \ll 1\right)$. При этом отношение моментов равно $c_{2} / c_{1}=0,1$. Видно, что в обоих случаях поведение кривых аналогично и значения нормы управления близки. С уменьшением отношения $c_{2} / c_{1}$ кривые, соответствующие точному и приближенному решениям, становятся все более близкими. Кроме того, стоит отметить, что зависимости нормы на рис. 1 и 2 имеют разный характер, как качественный, так и количественный. 


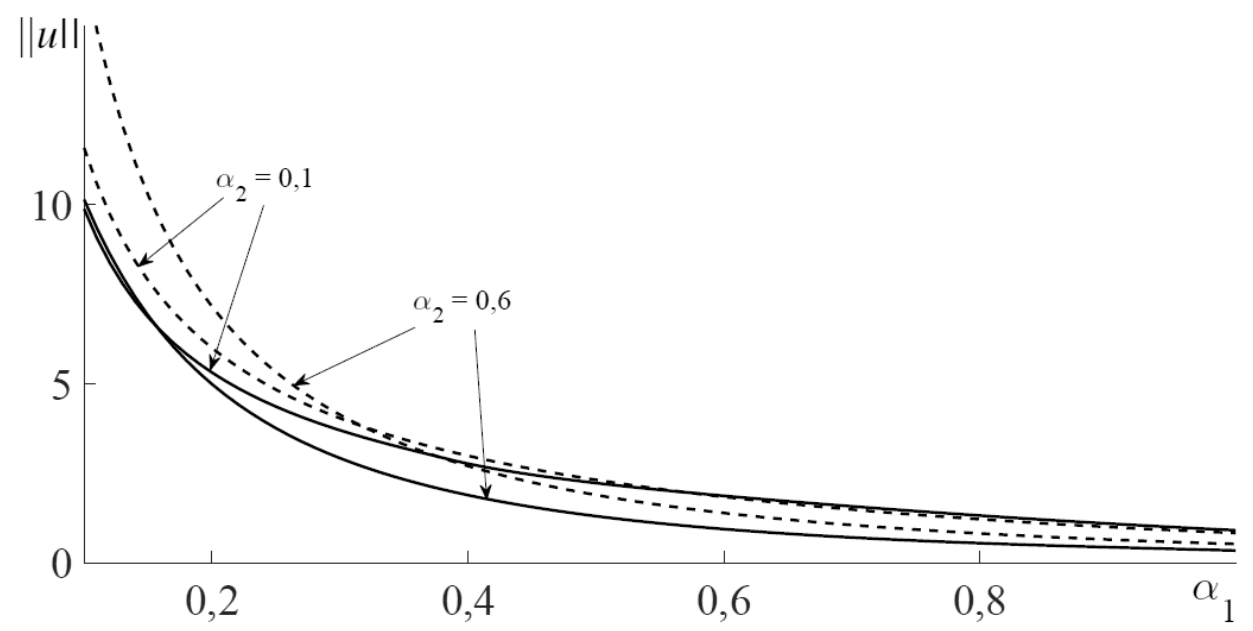

Рис. 1. Зависимости нормы управления от показателя $\alpha_{1}$ при фиксированном значении $\alpha_{2}$.

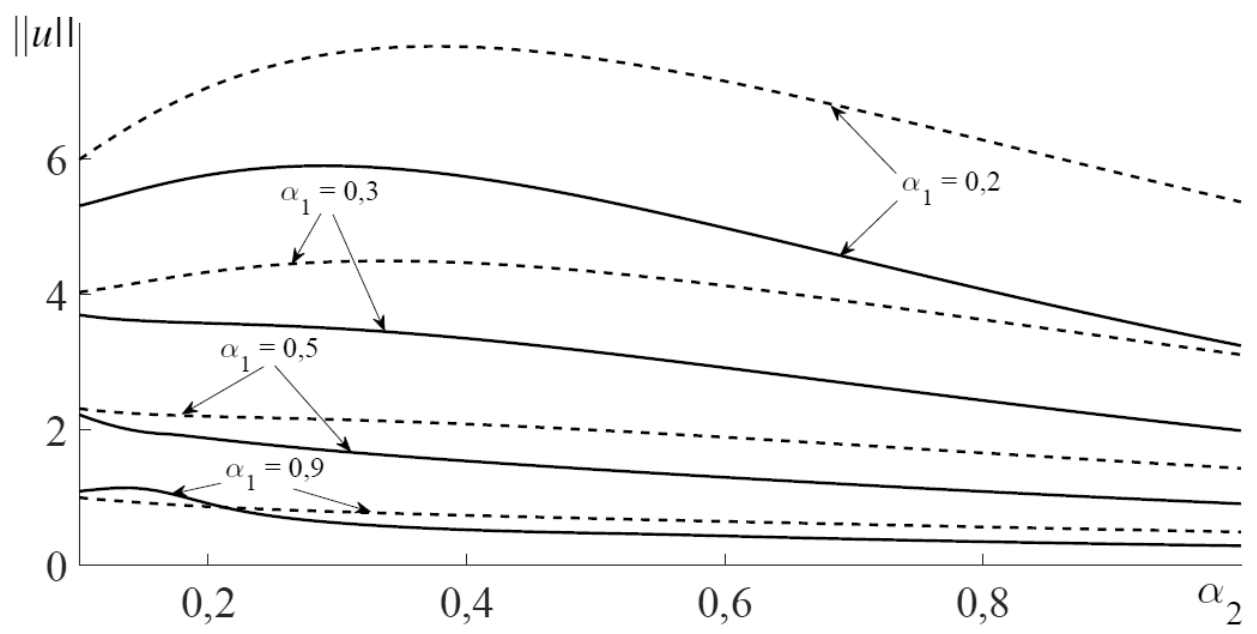

Рис. 2. Зависимости нормы управления от показателя $\alpha_{2}$ при фиксированном значении $\alpha_{1}$.

Интересной представляется оценка точности приближенных решений для случая $\alpha_{2} / \alpha_{1} \ll 1$. Ее можно характеризовать величиной относительной невязки норм оптимального управления:

$$
\eta=\frac{\lambda_{2}-\tilde{\lambda}_{2}}{\lambda_{2}} .
$$

На рис. 3 для системы Капуто представлены зависимости относительной невязки норм, вычисленных в случае, когда величина $\lambda_{2}$ вычисляется при $c_{2}=0$ точно (по формулам (7) и (23) при $\left.c_{2}=0\right)$, а величина $\tilde{\lambda}_{2}$ вычисляется по формулам (24) и (7). На рис. 4 аналогичные кривые приведены для случая, когда невязка вычисляется между нормами, вычисленными по формуле (24) с использованием для $\lambda_{2}$ оценки (23), а для $\tilde{\lambda}_{2}-$ оценки $(24)$ при $c_{2} / c_{1}=0,01$. Видно, что в обоих случаях величина невязки остается в пределах $10 \%$ для значений $\alpha_{2} \sim 0,2 \div 0,3$ при $\alpha_{1} \geqslant 0,8$.

Численное нахождение минимума функции (12) можно выполнять многими известными методами, в данной работе приведены результаты использования метода дихотомии. Алгоритм расчета был реализован как функция в среде MatLab, которая по входным данным рассчитывает коэффициенты, входящие в формулу (12), и определяет минимум искомой функции, выдавая массив 


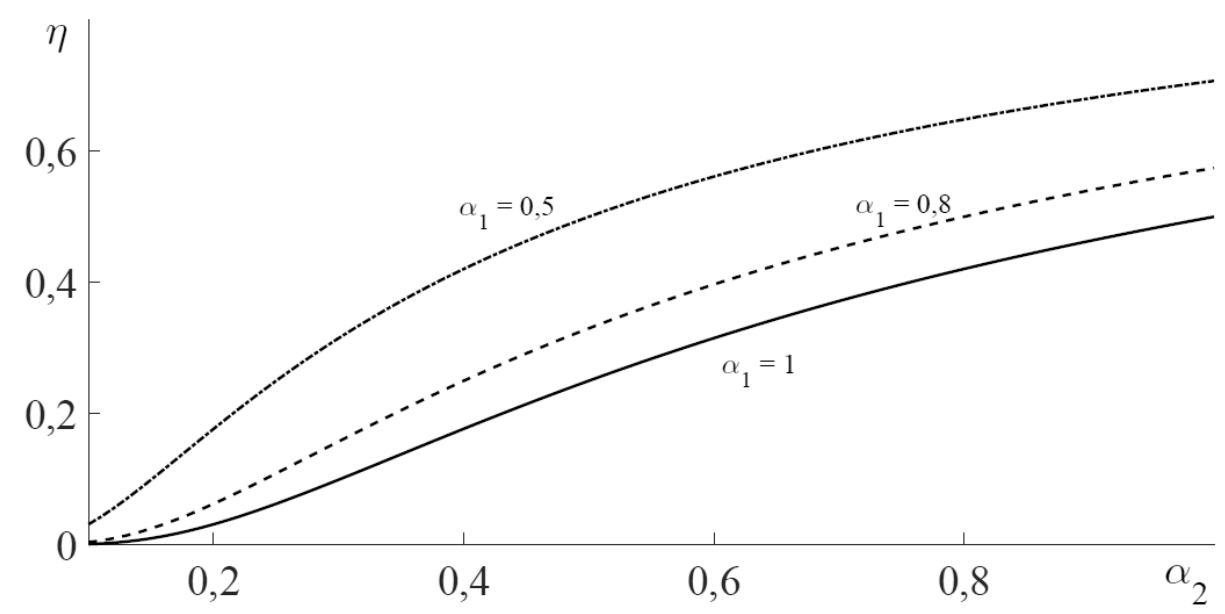

Рис. 3. Зависимость нормированной невязки норм управления от показателя $\alpha_{2}$ при $c_{2}=0$.

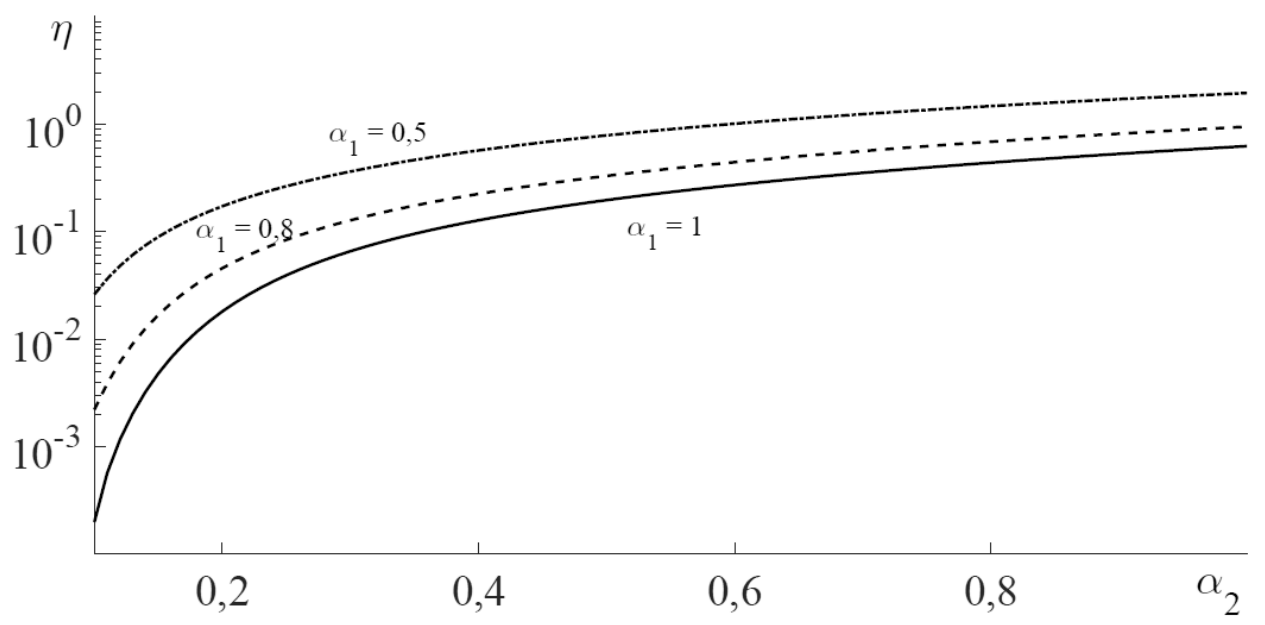

Рис. 4. Зависимость нормированной невязки норм управления от показателя $\alpha_{2}$ при $c_{2} / c_{1}=0.01$.

значений нормы управления и соответствующих им значений показателей дробного дифференцирования. При расчетах вначале проверялись условия теорем 1 и 2 и задавались интервалы поиска минимального значения функции согласно теореме 3. При расчете интервал поиска делился на 1000 точек. Такой способ вычисления уже позволяет находить решение в более общих случаях, когда моменты $c_{1}$ и $c_{2}$ оказываются соизмеримы или когда второй момент заметно превышает первый. На рис. 5 и 6 приведены зависимости нормы оптимального управления в случаях, когда отношение моментов $c_{2} / c_{1}$ равно 1 и 100 . При расчете задавались следующие значения параметров: $T=1, s_{1}^{0}=1, q_{2}^{T}=-1$ (для рис. 5$), q_{2}^{T}=-100$ (для рис. 6 ), значения остальных параметров выбирались как и выше.

Из представленных графиков (рис. 5 и 6) видно, что полученные зависимости квазимонотонны и не имеют особенностей. Интересной особенностью является то, что кривые на рис. 5 и 6 ведут себя противоположным образом: спадают в случае, когда моменты соизмеримы, как и в случае малого или нулевого отношения моментов (рис. 1), а при большом отношении моментов зависимости приобретают нарастающий характер. Кроме того, необходимо отметить, что во втором 


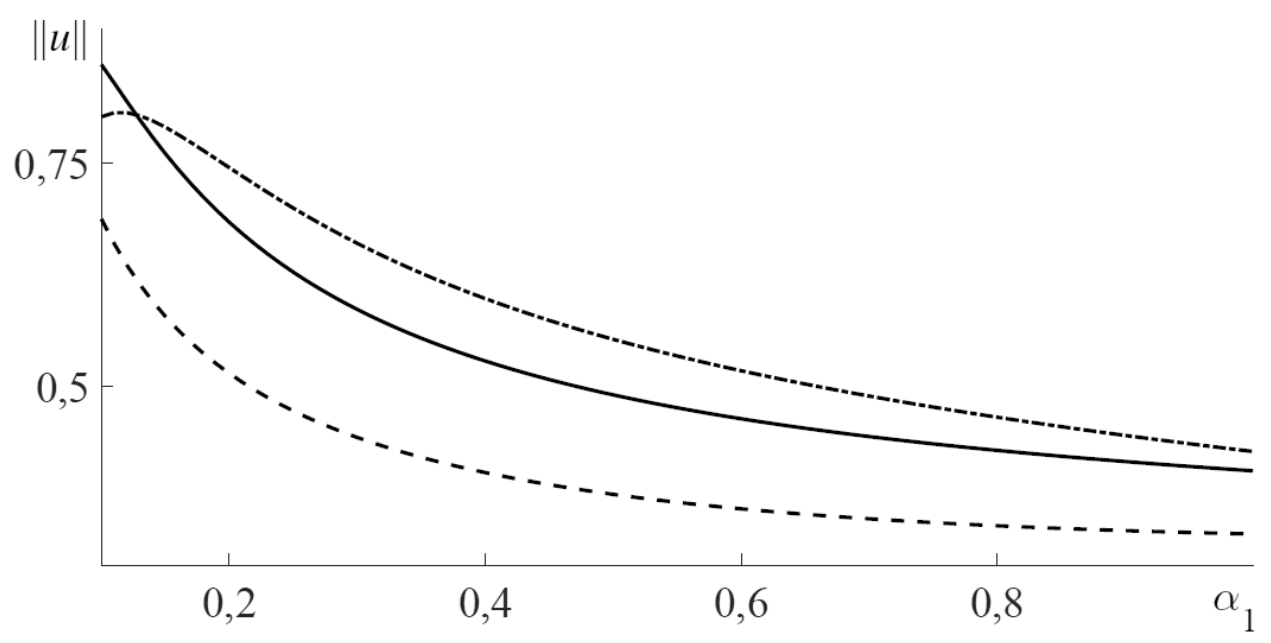

Рис. 5. Зависимости нормы управления от показателя $\alpha_{1}$ при фиксированном значении $\alpha_{2}=0,3$; 0,6; 0,9 (соответственно пунктирная, сплошная и штрихпунктирная кривые).

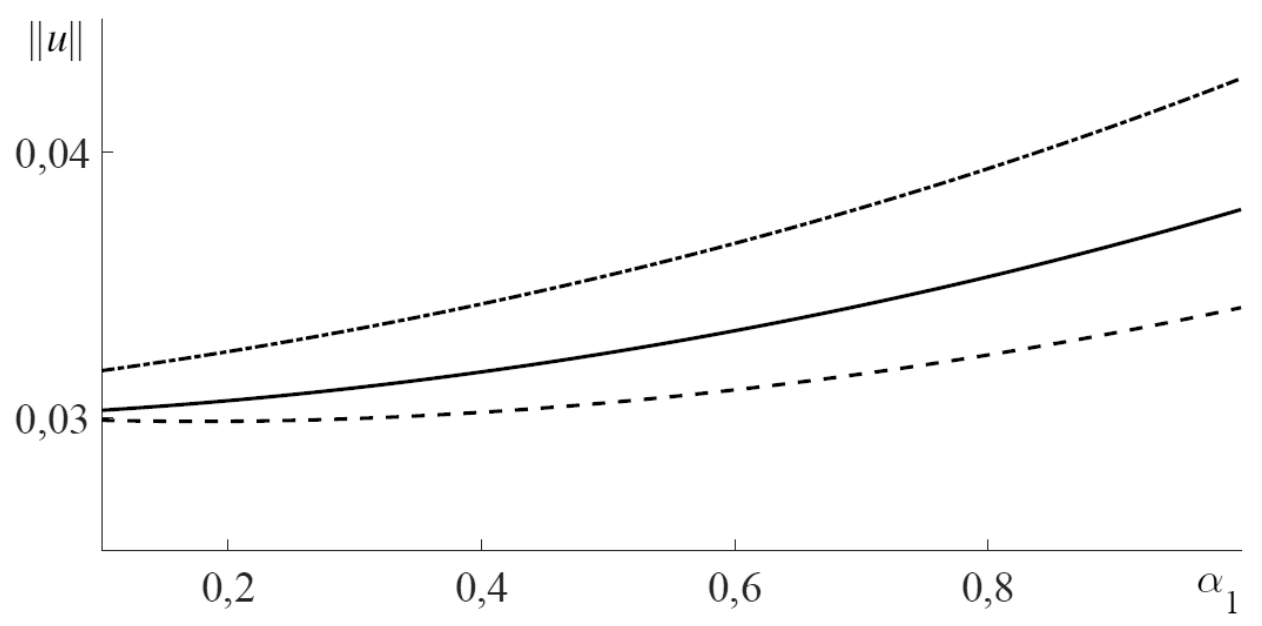

Рис. 6. Зависимости нормы управления от показателя $\alpha_{1}$ при фиксированном значении $\alpha_{2}=0,3$; 0,6; 0,9 (соответственно пунктирная, сплошная и штрихпунктирная кривые).

случае зависимости нормы от $\alpha_{1}$ и от $\alpha_{2}$ оказываются очень близки (визуально практически совпадают), чего не наблюдается в случае малого или близкого к 1 отношения моментов (см. выше, рис. 1 и 2 ).

7. Фазовые траектории двумерных систем дробного порядка на примере двойного интегратора. Анализ динамики двумерных систем дробного порядка с управлением, как и в случае систем целого порядка, включает две основные задачи: вычисление граничных траекторий, соответствующих граничным значениям управления, и оптимальных траекторий, соответствующих оптимальному управлению. Первый тип траекторий выделяет на фазовой плоскости область, содержащую все допустимые траектории системы, а второй тип траекторий демонстрирует динамику перехода системы в заданное состояние в режиме оптимального управления. Для систем целого порядка граничные траектории являются границами интегральной воронки соответствующего дифференциального включения, на основе которых строится фазовый портрет динамической системы с управлением [2]. 
Граничные траектории двойного интегратора можно вычислить, решив систему уравнений (1) при $u(t)= \pm l, l>0$. В результате для системы Хильфера получаются следующие выражения, представляющие собой временнь́е зависимости фазовых координат [8]:

$$
\begin{gathered}
q_{1}^{ \pm l}(t)=\frac{s_{1}^{0}\left(t-t_{0}\right)^{-\left(1-\alpha_{1}\right)\left(1-\mu_{1}\right)}}{\Gamma\left[1-\left(1-\alpha_{1}\right)\left(1-\mu_{1}\right)\right]}+\frac{s_{2}^{0}\left(t-t_{0}\right)^{\alpha_{1}-\left(1-\alpha_{2}\right)\left(1-\mu_{2}\right)}}{\Gamma\left[\alpha_{1}+1-\left(1-\alpha_{2}\right)\left(1-\mu_{2}\right)\right]} \pm \frac{l\left(t-t_{0}\right)^{\alpha_{1}+\alpha_{2}}}{\Gamma\left[\alpha_{1}+\alpha_{2}+1\right]} \\
q_{2}^{ \pm l}(t)=\frac{s_{2}^{0}\left(t-t_{0}\right)^{-\left(1-\alpha_{2}\right)\left(1-\mu_{2}\right)}}{\Gamma\left[1-\left(1-\alpha_{2}\right)\left(1-\mu_{2}\right)\right]} \pm \frac{l\left(t-t_{0}\right)^{\alpha_{2}}}{\Gamma\left[\alpha_{2}+1\right]}
\end{gathered}
$$

При вычислении граничных траекторий системы Адамара аналогичным образом можно получить следующие выражения [15]:

$$
\begin{gathered}
q_{1}^{ \pm l}(t)=\frac{s_{1}^{0}}{\Gamma\left(\alpha_{1}\right)}\left(\ln \frac{t}{t_{0}}\right)^{\alpha_{1}-1}+\frac{s_{2}^{0}}{\Gamma\left(\alpha_{1}+\alpha_{2}\right)}\left(\ln \frac{t}{t_{0}}\right)^{\alpha_{1}+\alpha_{2}-1} \pm \frac{l}{\Gamma\left[\alpha_{1}+\alpha_{2}+1\right]}\left(\ln \frac{t}{t_{0}}\right)^{\alpha_{1}+\alpha_{2}} \\
q_{2}^{ \pm l}(t)=\frac{s_{2}^{0}}{\Gamma\left(\alpha_{2}\right)}\left(\ln \frac{t}{t_{0}}\right)^{\alpha_{2}-1} \pm \frac{l}{\Gamma\left[\alpha_{2}+1\right]}\left(\ln \frac{t}{t_{0}}\right)^{\alpha_{2}}
\end{gathered}
$$

В общем случае в выражениях (26) и (27) не удается исключить время и явно выразить зависимость $q_{2}^{ \pm l}\left(q_{1}^{ \pm l}\right)$, описывающую поведение граничных траекторий системы, хотя это возможно в некоторых частных случаях $[8,15]$. Следует также отметить, что при $\alpha_{1}=\alpha_{2}=1$ выражения (26) и (27) могут быть преобразованы к одному и тому же квадратному уравнению, которое описывает граничные траектории двойного интегратора целого порядка [2].

Поведение граничных траекторий проиллюстрировано на рис. 7-10 для случая $c_{2}=0, T=10$, $s_{1}^{0}=1, s_{2}^{0}=0, l=1, t_{0}=0$ (для системы Хильфера) или $t_{0}=1$ (для системы Адамара). Видно, что для этих траекторий характерны две основные особенности. Во-первых, траектории системы дробного порядка лежат внутри области, ограничиваемой траекториями системы целого порядка. Т. е., область, содержащая допустимые траектории системы дробного порядка оказывается у́же, чем у такой же системы целого порядка. При этом наиболее узкой эта область оказывается для системы Адамара [15]. Во-вторых, имеется качественное отличие в поведении траекторий в случаях $\alpha_{1}=1$ и $\alpha_{1} \neq 1$, т. е. когда связь между фазовыми координатами является «классической» (импульс есть производная координаты) или «неклассической». В последнем случае образуется клиновидная область, сужающаяся и вытягивающаяся вдоль оси абсцисс с уменьшением параметров $\mu_{1,2}$ оператора Хильфера (см. рис. 11). Аналогичная картина наблюдается для двойного интегратора Адамара с уменьшением параметра $\alpha_{2}[15]$.

Фазовые траектории двойного интегратора, соответствующие режиму оптимального управления, можно вычислить, подставив полученные выше выражения для оптимального управления в систему (1) и решив ее в этом случае. Тогда оптимальные траектории системы Хильфера будут описываться выражениями [8]:

$$
\begin{aligned}
q_{1}(t)= & \frac{s_{1}^{0}\left(t-t_{0}\right)^{-\left(1-\alpha_{1}\right)\left(1-\mu_{1}\right)}}{\Gamma\left[1-\left(1-\alpha_{1}\right)\left(1-\mu_{1}\right)\right]}+\frac{s_{2}^{0}\left(t-t_{0}\right)^{\alpha_{1}-\left(1-\alpha_{2}\right)\left(1-\mu_{2}\right)}}{\Gamma\left[\alpha_{1}+1-\left(1-\alpha_{2}\right)\left(1-\mu_{2}\right)\right]}+ \\
& \quad+\frac{\lambda_{2}}{\Gamma\left(\alpha_{1}+\alpha_{2}+1\right)}\left[\left(t-t_{0}\right)^{\alpha_{1}+\alpha_{2}}-2\left(t-t^{\prime}\right)^{\alpha_{1}+\alpha_{2}} \Theta\left(t-t^{\prime}\right)\right], \\
q_{2}(t)= & \frac{s_{2}^{0}\left(t-t_{0}\right)^{-\left(1-\alpha_{2}\right)\left(1-\mu_{2}\right)}}{\Gamma\left[1-\left(1-\alpha_{2}\right)\left(1-\mu_{2}\right)\right]}+\frac{\lambda_{2}}{\Gamma\left(\alpha_{2}+1\right)}\left[\left(t-t_{0}\right)^{\alpha_{2}}-2\left(t-t^{\prime}\right)^{\alpha_{2}} \Theta\left(t-t^{\prime}\right)\right],
\end{aligned}
$$

где $t^{\prime}$ определяется формулой $(10), \Theta\left(t-t^{\prime}\right)$ - функция Хевисайда. 


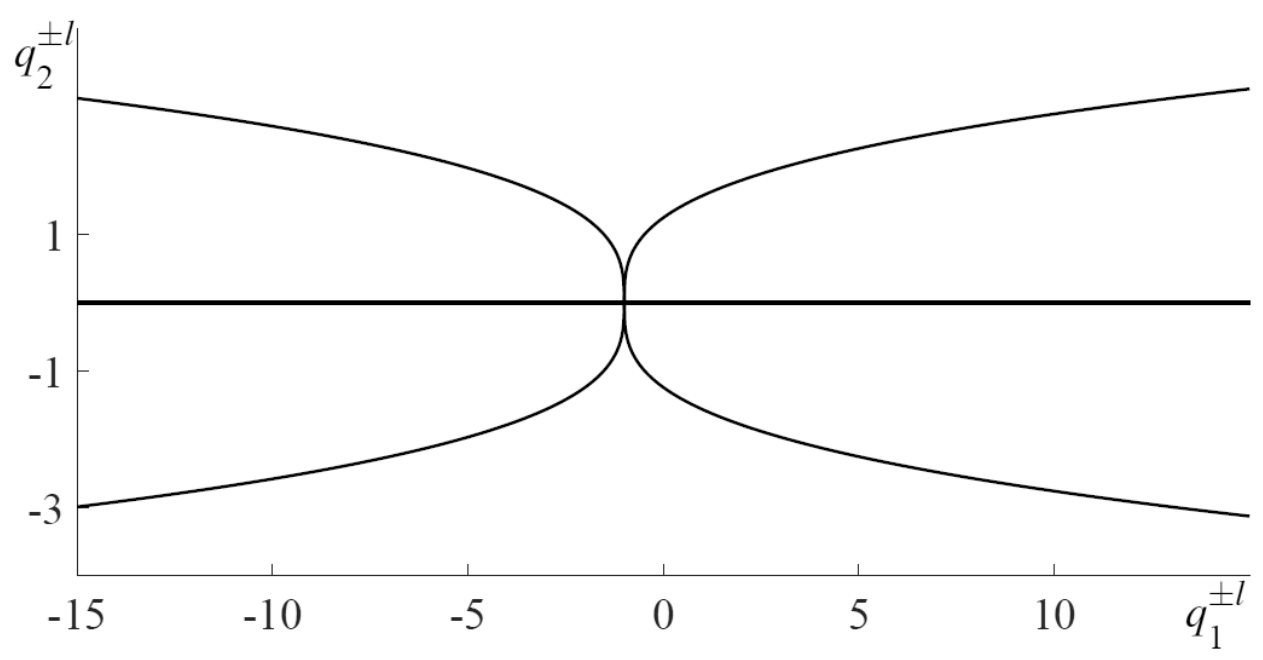

Рис. 7. Граничные траектории двойного интегратора Хильфера в случае $\alpha_{1}=1, \alpha_{2}=0,5$, $\mu_{2}=0,7$.

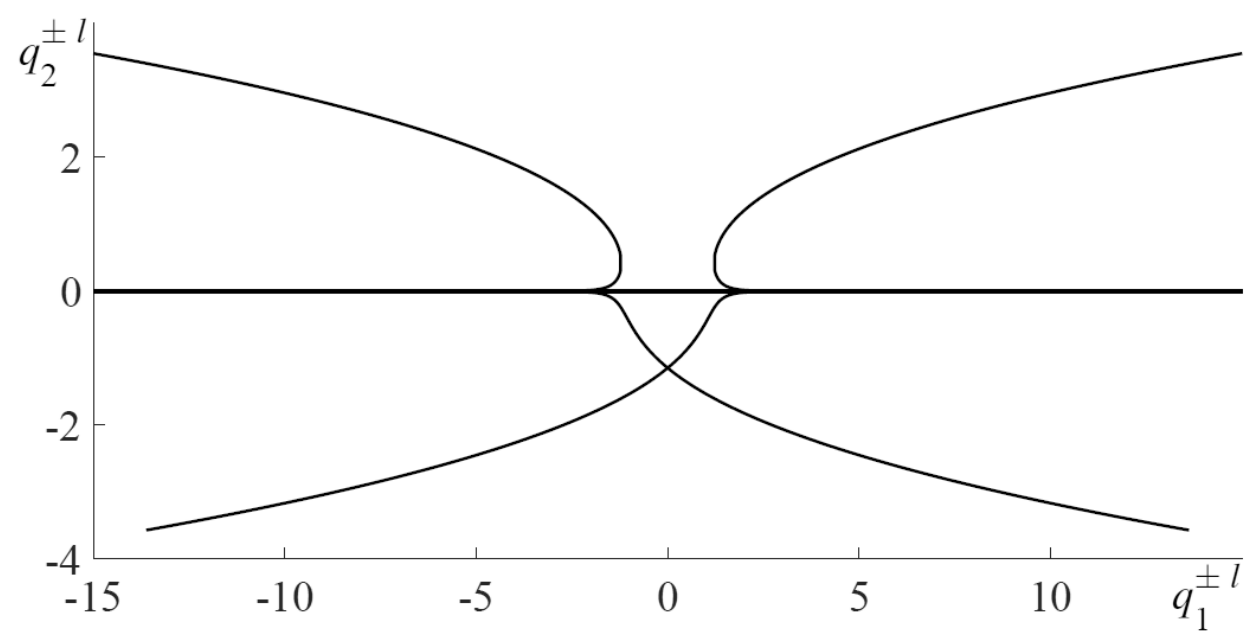

Рис. 8. Граничные траектории двойного интегратора Хильфера в случае $\alpha_{1}=0,7, \alpha_{2}=0,5$, $\mu_{1}=\mu_{2}=0,7$.

Аналогично, оптимальные траектории системы Адамара описываются выражениями [15]:

$$
\begin{aligned}
q_{1}(t)= & \frac{s_{1}^{0}}{\Gamma\left(\alpha_{1}\right)}\left(\ln \frac{t}{t_{0}}\right)^{\alpha_{1}-1}+\frac{s_{2}^{0}}{\Gamma\left(\alpha_{1}+\alpha_{2}\right)}\left(\ln \frac{t}{t_{0}}\right)^{\alpha_{1}+\alpha_{2}-1}+ \\
& +\lambda_{2}\left[\left(\ln \frac{t}{t_{0}}\right)^{\alpha_{1}+\alpha_{2}}-2\left(\ln \frac{t}{t^{\prime}}\right)^{\alpha_{1}+\alpha_{2}} \Theta\left(t-t^{\prime}\right)\right], \\
q_{2}^{ \pm l}(t)= & \frac{s_{2}^{0}}{\Gamma\left(\alpha_{2}\right)}\left(\ln \frac{t}{t_{0}}\right)^{\alpha_{2}-1}+\frac{\lambda_{2}}{\Gamma\left(\alpha_{2}+1\right)}\left[\left(\ln \frac{t}{t_{0}}\right)^{\alpha_{2}}-2\left(\ln \frac{t}{t^{\prime}}\right)^{\alpha_{2}} \Theta\left(t-t^{\prime}\right)\right],
\end{aligned}
$$

где значение $t^{\prime}$ определяется формулой (11). 


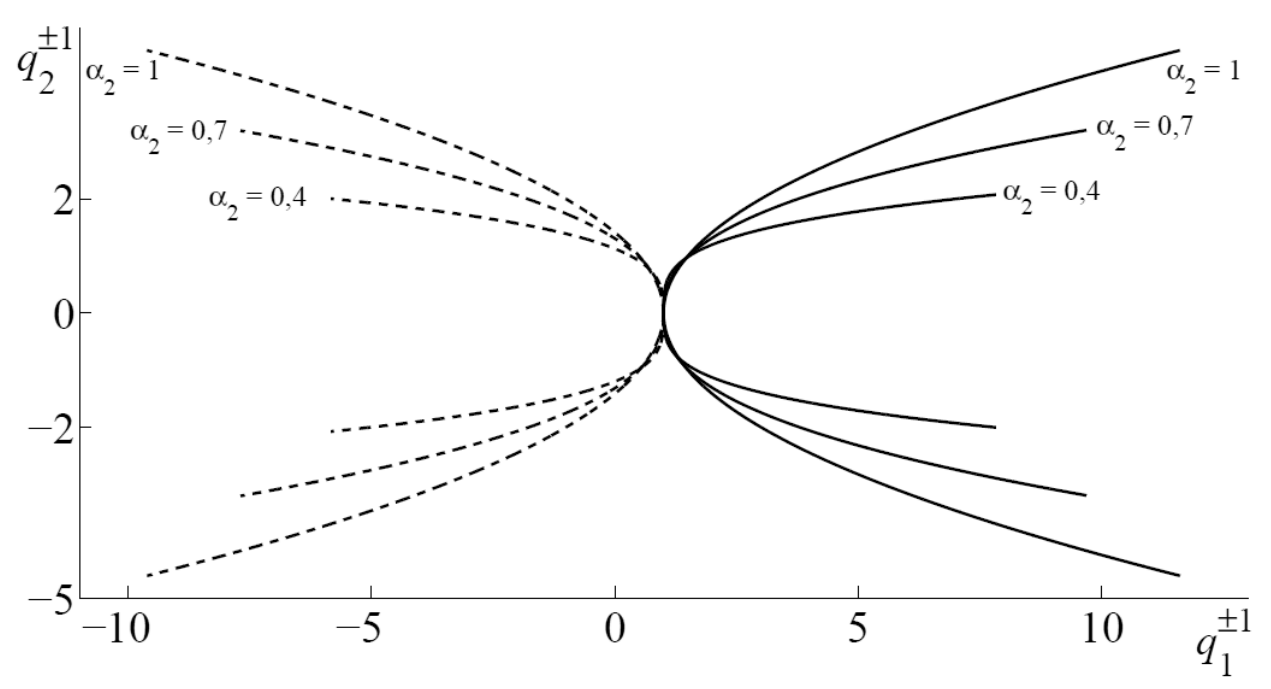

Рис. 9. Граничные траектории двойного интегратора Адамара в случае $\alpha_{1}=1$, при разных значениях показателя $\alpha_{2}$.

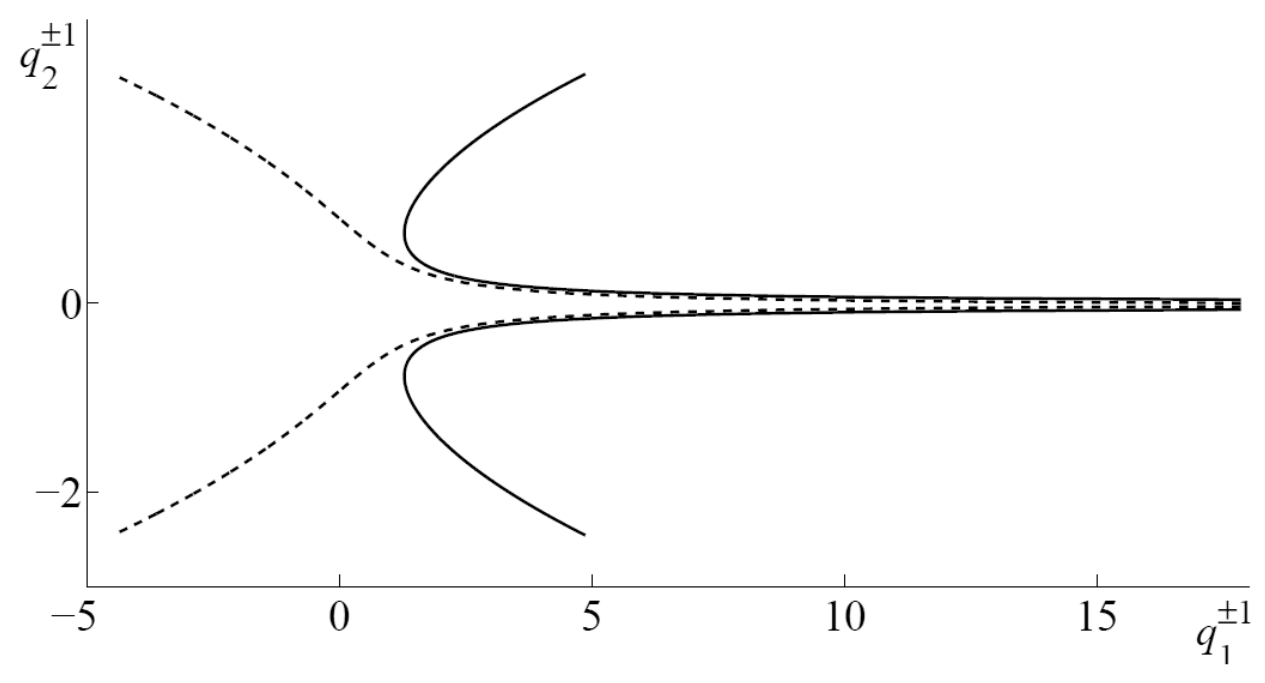

Рис. 10. Граничные траектории двойного интегратора Адамара в случае $\alpha_{1}=0,5, \alpha_{2}=0,5$.

Рассмотрим далее случай $c_{2}=0$, для которого оптимальное управление дается формулами (17) и (18), а норма оптимального управления дается выражением:

$$
\lambda_{2}=\frac{\Gamma\left(\alpha_{1}+\alpha_{2}+1\right)}{1-2^{-\alpha_{1} / \alpha_{2}}} \frac{\left|c_{1}\right|}{\left(T-t_{0}\right)^{\alpha_{1}+\alpha_{2}}}
$$

для системы Хильфера [8] и

$$
\lambda_{2}=\frac{\Gamma\left(\alpha_{1}+\alpha_{2}+1\right)}{1-2^{-\alpha_{1} / \alpha_{2}}} \frac{\left|c_{1}\right|}{\left(\ln \frac{t}{t_{0}}\right)^{\alpha_{1}+\alpha_{2}}}
$$

для системы Адамара [15]. Примеры оптимальных фазовых траекторий системы Адамара показаны на рис. 12 и 13 [15]. При вычислении выбирались те же значения параметров, что и выше (при расчете граничных траекторий). Видно, что, как и для граничных траекторий, случаи $\alpha_{1}=1$ и $\alpha_{1} \neq 1$ различаются качественно. В первом случае при изменении второго показателя 


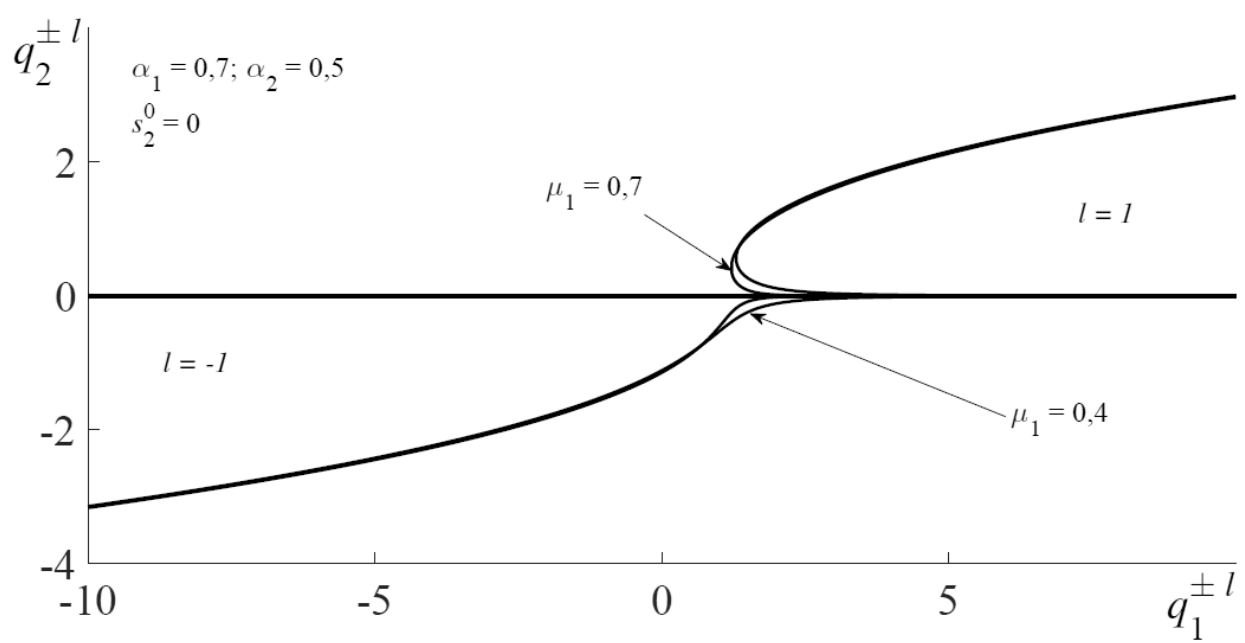

Рис. 11. Граничные траектории двойного интегратора Хильфера в случае $\alpha_{1}=0,7, \alpha_{2}=0,5$, $m u_{2}=0,7$ при различных значениях параметра $\mu_{1}$.

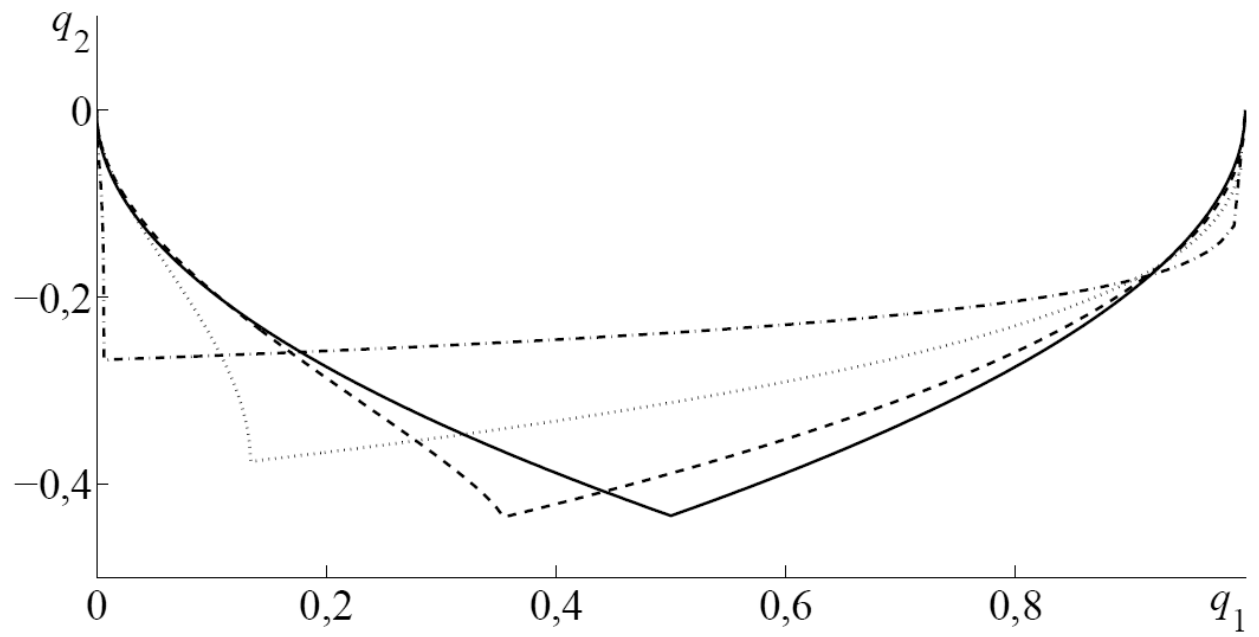

Рис. 12. Оптимальные траектории двойного интегратора Адамара в случае $c_{2}=0, \alpha_{1}=1$, при разных значениях показателя $\alpha_{2}$.

дробного дифференцирования имеет место только смещение точки переключения оптимального управления. Во втором же случае наблюдается эффект перерегулирования: траектория выходит за пределы отрезка, соединяющего начальное и конечное состояния, совершает переключение вне этого отрезка и «возвращается» в конечное состояние. При этом эффект выражен тем сильнее, чем меньше показатель дробного дифференцирования в первом звене. Аналогичная ситуация наблюдается и для системы Хильфера. При этом эффект перерегулирования усиливается еще и с ростом параметра $\mu_{1}$, что соответствует отмеченному ранее факту о более сильном проявлении перерегулирования для систем Капуто, чем для систем Римана-Лиувилля [8]. Следует также отметить, что эффект перерегулирования сильнее выражен для системы Адамара, чем для системы Римана-Лиувилля [15]. 


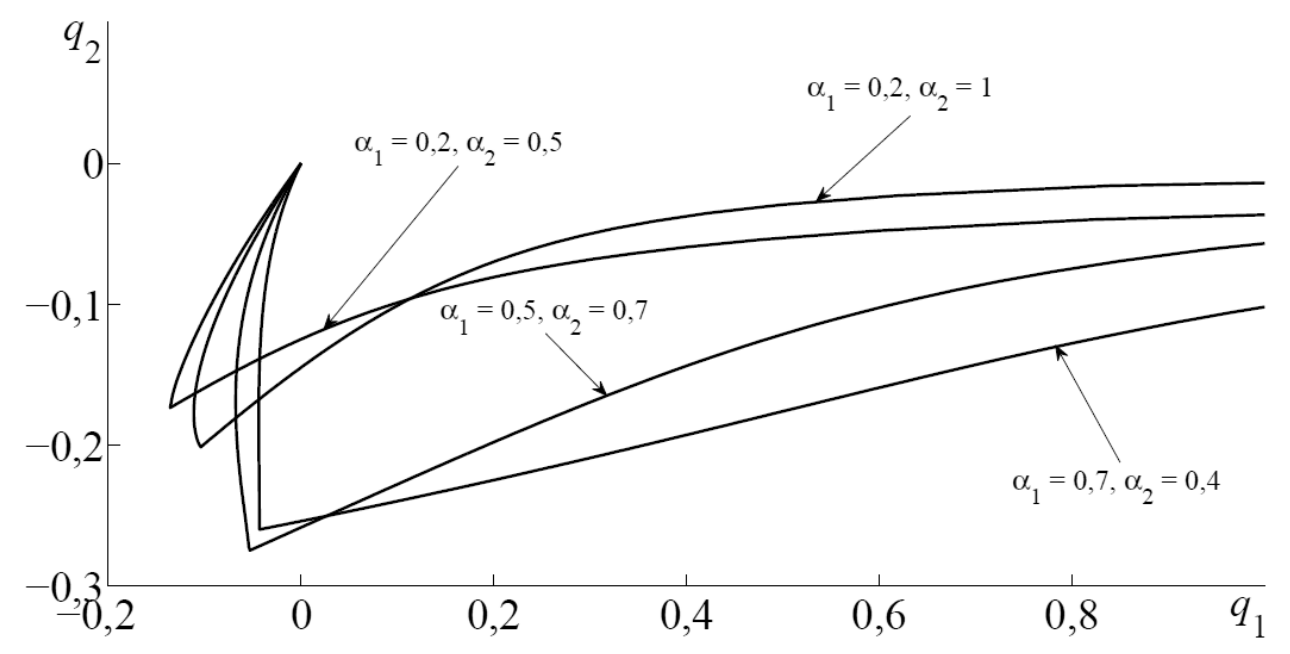

Рис. 13. Оптимальные траектории двойного интегратора Адамара в случае $c_{2}=0$ при разных значениях показателей $\alpha_{1}$ и $\alpha_{2}$.

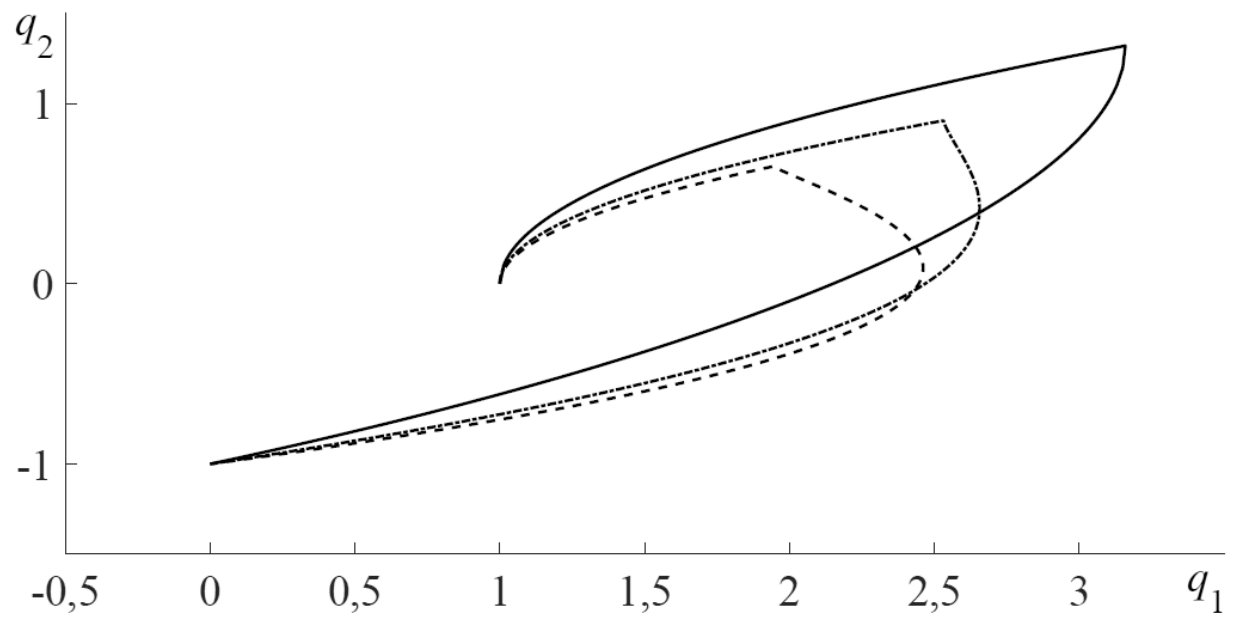

Рис. 14. Оптимальные траектории двойного интегратора Хильфера в случае $c_{2} \neq 0, \alpha_{1}=\alpha_{2}=\alpha$ при $\mu_{1}=1$ и разных значениях показателей $\alpha$ и $\mu_{2}: \alpha=0,3, \mu_{2}=1$ (сплошная линия), $\alpha=0,9$, $\mu_{2}=0,8$ (пунктирная линия), $\alpha=0,7, \mu_{2}=0,3$ (штрихпунктирная линия).

На рис. 14 показаны оптимальные траектории системы Хильфера для случая $c_{2} \neq 0, \alpha_{1}=$ $\alpha_{2}=\alpha$ (норма оптимального управления в данном случае вычисляется по формуле (19), а оптимальное управление определяется выражением (17)). Для расчета были выбраны следующие значения параметров: $s_{1}^{0}=1, s_{2}^{0}=0, q_{1}^{T}=0, q_{2}^{T}=-1, \mu_{1}=1, t_{0}=0, T=10$. Здесь также заметен эффект перерегулирования, степень проявления которого зависит от параметров оператора дробного дифференцирования.

Таким образом, несмотря на возможные количественные различия в значениях фазовых координат и управления, представленные выше результаты говорят о наличии весьма схожих особенностей качественной динамики систем, описываемых уравнениями с разным типом оператора дробного дифференцирования. 
8. Дополнительные замечания о фазовых траекториях маятника дробного порядка. В качестве другого примера двумерной системы дробного порядка можно рассмотреть маятник:

$$
\begin{aligned}
& t_{0} D_{t}^{\rho_{1}} q_{1}(t)=q_{2}(t), \\
& t_{0} D_{t}^{\rho_{2}} q_{2}(t)=-q_{1}(t)+u(t) .
\end{aligned}
$$

В работе [4] было получено решение задачи оптимального управления системой (30) в случае, когда оператор дробного дифференцирования понимается в смысле Капуто (т. е., оператор Хильфера при $\mu=1$ ). При этом полученное решение оказалось представимо непрерывной функцией времени и оптимальным в смысле минимума нормы в пространстве $L_{2}[0, T]$. На основе этого решения также были вычислены граничные и оптимальные фазовые траектории системы и было показано, что граничные траектории, как и в случае двойного интегратора, выделяют подобласть в области, ограниченной граничными траекториями системы целого порядка.

9. Заключение. В работе получен ряд результатов в исследовании задачи оптимального управления двойным интегратором дробного порядка в случае, когда допустимые управления являются существенно ограниченными функциями. Поставленная задача сведена к задаче минимизации некоторой функции одной переменной и обоснованы условия, при которых эта задача минимизации имеет решение, а также определены области локализации решений.

Построено точное аналитическое решение для случая, когда показатели обоих звеньев интегратора равны. Получены приближенные решения задачи в случаях, когда либо отношение моментов $c_{2} / c_{1}$, либо отношение показателей дробного дифференцирования $\alpha_{2} / \alpha_{1}$ являются малым параметром. Также приведены результаты численного решения задачи при произвольном значении отношения моментов и показателей дробного дифференцирования.

Все полученные результаты применимы для систем, которые описываются в терминах операторов Хильфера (включая как предельные случаи операторы Капуто и Римана-Лиувилля) и Адамара.

Исследованы вопросы качественной динамики изучаемых систем. Проведено сравнение граничных и оптимальных траекторий двумерных систем дробного порядка, описываемых уравнениями с разным типом оператора дробного дифференцирования. Показано, что в поведении всех этих систем есть общие свойства: граничные траектории систем дробного порядка ограничивают область допустимых траекторий системы внутри таковой области соответствующих систем целого порядка. Кроме того, для двойного интегратора поведение системы качественно отличается в случаях, когда показатель дробного дифференцирования в первом звене интегратора равен 1 и отличен от нее. В последнем случае проявляется эффект перерегулирования, выраженность которого зависит от параметров операторов дробного дифференцирования, входящих в уравнения динамики системы.

\section{СПИСОК ЛИТЕРАТУРЫ}

1. Бутковский А. Г. Методы управления системами с распределенными параметрами. - М.: Наука, 1975.

2. Бутковский А. Г. Фазовые портреты управляемых динамических систем. - М.: Наука, 1985.

3. Крейн М. Г., Нудельман А. А. Проблема моментов Маркова и экстремальные задачи. - М.: Наука, 1973.

4. Кубышкин B. А., Постнов С. С. Исследование двух задач оптимального управления маятником дробного порядка с помощью метода моментов// Пробл. управл. - 2014. - № 3. - C. 14--22.

5. Кубышкин B. А., Постнов C. С. Задача оптимального управления линейной стационарной системой дробного порядка в форме проблемы моментов: постановка и исследование// Автомат. телемех. 2014. - № 5. - С. 3-17.

6. Постнов С. С. Исследование задачи оптимального управления для одиночного и двойного интеграторов дробного порядка с помощью метода моментов// Пробл. управл. -2012 . - № 5. - С. 9-17.

7. Постнов С. С. Задачи оптимального управления для линейных систем дробного порядка, заданных уравнениями с производной Адамара// Докл. РАН. - 2017. - 476, № 2. - С. 143-147.

8. Постнов C. С. Задачи оптимального управления для некоторых линейных систем дробного порядка, заданных уравнениями с производной Хильфера// Пробл. управл. - 2018. - № 5. - С. 14-25. 
9. Постнова E. A. Оптимальное управление движением системы, моделируемой двойным интегратором дробного порядка// Пробл. управл. - 2018. - № 2. - С. 40-46.

10. Bai J., Wen G., Rahmani A., Yu Y. Consensus for the fractional-order double integrator multi-agent systems based on the sliding mode estimator// IET Control Theory Appl. — 2018. — 12, № 5. — P. 621-628.

11. Hilfer R. Fractional time evolution// in: Applications of Fractional Calculus in Physics. — Singapore: World Scientific, 2000. - P. 87--130.

12. Kamal S., Raman A., Bandyopadhyay B. Finite-time stabilization of fractional-order uncertain chain of integrator: An integral sliding mode approach// IEEE Trans. Automat. Control. — 2013. — 58, № 6. — P. 1597-1602.

13. Kilbas A. A., Srivastava H. M., Trujillo J. J. Theory and Applications of Fractional Differential Equations. - Amsterdam: Elsevier, 2006.

14. Mozyrska D., Torres D. F. M. Modified optimal energy and initial memory of fractional continuous-time linear systems// Signal Process. — 2011. — 91, № 3. — P. 379-385.

15. Postnov S. Optimal control problem for linear fractional-order systems, described by equations with Hadamard-type derivative// J. Phys. Conf. Ser. — 2017. — 918, № 012026.

16. Tricaud C., Chen Y. Q. Time-optimal control of systems with fractional dynamics// Int. J. Differ. Equations. - 2010. - 2010, № 461048 .

Постнов Сергей Сергеевич

Институт проблем управления им. В. А. Трапезникова РАН, Москва

E-mail: postnov.sergey@inbox.ru

Постнова Елена Александровна

Институт проблем управления им. В. А. Трапезникова РАН, Москва

E-mail: postnova@ipu.ru 